\title{
Targeting the autonomic nervous system during AF ablation: should we fight or take flight?
}

\author{
Tolga Aksu and Dhiraj Gupta ${ }^{2}$ \\ ${ }^{1}$ Kocaeli Derince Egitim ve Arastirma Hastanesi \\ ${ }^{2}$ University of Liverpool Faculty of Health and Life Sciences
}

December 21, 2020

\begin{abstract}
Demonstration that the myocardial sleeves of the pulmonary veins (PVs) are the main triggering and maintaining foci for paroxysmal atrial fibrillation (AF) have stimulated studies investigating electrophysiological properties of PVs and the adjacent left atrial (LA) myocardium. It has been shown that PV myocytes have a shorter action potential duration and are more prone to effects of local autonomic nerve stimulation in terms of shortening of action potential duration, early after depolarization formation and triggered firing compared to left atrial myocytes (1). The intrinsic cardiac autonomic nervous system (ICANS) forms clusters of neurons called ganglionic plexi (GPs), and studies using histologic examination of heart sections have shown that these GPs are localized preferentially at certain epicardial sites adjacent to the left and right atria (2). The precise role of ICANS in AF continues to be an area of intense research (3), and matters are not helped by the uncertainty regarding the best way to identify and target ICANS peri-procedurally. As there can be significant variability of GP sites in individual patients, endocardial high-frequency stimulation (HFS) has been used to aid their localization in the electrophysiology laboratory (4).
\end{abstract}

Targeting the autonomic nervous system during AF ablation: should we fight or take flight?

Tolga Aksu ${ }^{1}$

Dhiraj Gupta ${ }^{2}$, MD, FRCP

1

${ }^{2}$ Liverpool Heart and Chest Hospital, University of Liverpool, UK

Word count : 1142 (including table and table legend, excluding references)

Key words: Atrial fibrillation, Catheter ablation, Ganglionic plexus, autonomic nervous system

Funding: None

Correspondence

Name: Professor Dhiraj Gupta, MD https://orcid.org/0000-0002-3490-090X

Address: Department of Cardiology, Liverpool Heart and Chest Hospital, UK L14 3PE

Phone: +44 151600 1793; Fax: +44 1516001696

Email: Dhiraj.gupta@lhch.nhs.uk

Demonstration that the myocardial sleeves of the pulmonary veins (PVs) are the main triggering and maintaining foci for paroxysmal atrial fibrillation (AF) have stimulated studies investigating electrophysiological properties of PVs and the adjacent left atrial (LA) myocardium. It has been shown that PV myocytes have a 
shorter action potential duration and are more prone to effects of local autonomic nerve stimulation in terms of shortening of action potential duration, early after depolarization formation and triggered firing compared to LA myocytes (1). The intrinsic cardiac autonomic nervous system (ICANS) forms clusters of neurons, ganglionic plexi (GP), and studies using histologic examination of heart sections have shown that these GPs are localized preferentially at certain epicardial sites adjacent to the left and right atria (2). The precise role of targeting ICANS in AF ablation continues to be an area of intense research (3), and matters are not helped by the uncertainty regarding the best way to identify and target ICANS peri-procedurally. As there can be significant variability of GP sites in individual patients, endocardial high-frequency stimulation (HFS) has been used to aid their localization in the electrophysiology laboratory (4). Although HFS stimulation protocols have varied significantly between studies, these mostly involve delivering electrical pulses with a frequency of $20 \mathrm{~Hz}$, amplitude of $0.1-1.0 \mathrm{~mA}$, and pulse duration of 1-10 msec at each site using specially developed stimulators $(3,4)$. The first $2-4$ seconds HFS mostly causes parasympathetic effects as an immediate response, whilst a longer application of 8-10 seconds stimulates a delayed sympathetic response (4). Demonstration of a [?]50\% increase in mean R-R interval during AF, often accompanied by hypotension, is accepted as a significant parasympathetic response and serves as a surrogate marker of GP location.

In this issue of the journal, Sandler et al (5) present the results of a pilot study in patients with paroxysmal $\mathrm{AF}$ who were randomized to selective endocardial ablation of GPs or to conventional circumferential PV isolation. The investigators delivered synchronized HFS from multiple evenly distributed LA sites (103 \pm 28 sites per patient), and ablated those sites where HFS induced atrial ectopy, atrial tachycardia or AF, or where significant parasympathetic effects were elicited ( $21 \pm 10$ sites per patient). 67 patients were randomized to GP ablation $(n=39)$ or PV isolation $(n=28)$, although eight of 39 patients initially randomized to GP were crossed-over to the PV isolation group due to sustained AF precluding completion of GP mapping protocol. At the end of 12 months follow-up, $61 \%$ patients with PV isolation and $49 \%$ patients with GP ablation were free from $\mathrm{AF} /$ atrial tachycardia $(\mathrm{p}=0.27)$. The amount of $\mathrm{RF}$ energy delivered in the GP group was significantly lower, although this did not translate to a lower procedure time (mean 3.7 hours) or in a lower complication rate. The authors should be congratulated for the bold study design in which they were prepared to leave patients in the GP group without PV isolation to test their hypothesis. The randomised double-blind study design is another strength. On the flip side, readers will note the modest 12 -month success rates of around $50 \%$ seen in both groups in spite of limited ECG monitoring; these are in all likelihood related to the time period when the procedures were performed (2013-2017), before the advent of composite ablation indices and before the current emphasis on lesion contiguity that has enabled much higher single procedure success rates with PVI for paroxysmal AF. Whether the outcomes of a GP based ablation strategy would have been higher with these modern tools is a moot point. We are also left with several unanswered questions. Were the GP sites preferentially located at certain LA regions, why were right atrial sites not tested, what effect did general anaesthesia have on the reliability of GP identification, and importantly, would a third group, that of PV isolation plus GP ablation, have had higher success rates than either strategy alone? Also, given that the mean heart rates of patients in the GP group on serial Holter monitoring at follow up were no different to those in the PVI group, one also wonders how effective ablation actually was in terms of modifying the ICANS. Hopefully, the planned larger randomised study by the same group, NCT02487654, would answer some of these questions.

\section{What have other studies shown?}

Several groups have previously evaluated the clinical impact of ICANS modulation on AF, both endocardially with catheter ablation as well as epicardially with surgical ablation (Table 1). Although some studies have shown benefit of adding ICANS modulation to PV isolation $(6-8,10)$, others have not $(9,11)$. Certainly, no study has shown ICANS modulation alone to be superior to PVI. Furthermore, it is important to note that ICANS modulation can be associated with procedural risks, either in terms of procedural complications (11) or greater radiation exposure (10). Indeed, an apparently paradoxical interaction between ICANS and AF has been reported too; in a group of dogs in whom the major GPs and ligament of Marshall were ablated, significant prolongation of the effective refractory period and decrease in AF inducibility was seen acutely, and yet eight weeks later, the effective refractory period was significantly shorter and AF inducibility was 
significantly greater than in a sham control group (12).

\section{What next?}

As shown in the table, published data to date do not support ICANS modulation as an alternative to PV isolation. This is especially as ablation technologies have made the latter a streamlined, safe procedure with unequivocal objective end-points and excellent clinical results. Whether ICANS modulation would have a role in carefully selected subgroups, such as those with AF recurrence in spite of documented isolated PVs, remains to be proven. As of now, we believe that when it comes to the ablating the autonomic nervous system in AF, it may be preferable to take flight rather than to fight.

\section{References}

1. Patterson E, Po SS, Scherlag BJ, Lazzara R. Triggered firing in pulmonary veins initiated by in vitro autonomic nerve stimulation. Heart Rhythm 2005;2(6):624-31

2. Armour JA, Murphy DA, Yuan BX, Macdonald S, Hopkins DA. Gross and microscopic anatomy of the human intrinsic cardiac nervous system. Anat Rec. 1997;247:289-98

3. Aksu T, Güler TE, Mutluer FO, Oto MA. Vagal denervation in atrial fibrillation ablation: A comprehensive review. Anatol J Cardiol. 2017;18:142-148

4. Po SS, Nakagawa H, Jackman WM. Localization of left atrial ganglionated plexi in patients with atrial fibrillation. J Cardiovasc Electrophysiol. 2009;20:1186-1189

5. Sandler et al. J Cardiovasc Electrophysiol (in press)

6. Pokushalov E, Romanov A, Shugayev P, Artyomenko S, Shirokova N, Turov A, Katritsis DG. Selective ganglionated plexi ablation for paroxysmal atrial fibrillation. Heart Rhythm 2009;6:1257-64

7. Katritsis DG, Giazitzoglou E, Zografos T, Pokushalov E, Po SS, Camm AJ. Rapid pulmonary vein isolation combined with autonomic ganglia modification: a randomized study. Heart Rhythm. 2011;8:672-8

8. Katritsis DG, Pokushalov E, Romanov A, Giazitzoglou E, Siontis GC, Po SS, Camm AJ, Ioannidis JP. Autonomic denervation added to pulmonary vein isolation for paroxysmal atrial fibrillation: a randomized clinical trial. J Am Coll Cardiol. 2013;62:2318-25

9. Pokushalov E, Romanov A, Katritsis DG, Artyomenko S, Shirokova N, Karaskov A, Mittal S, Steinberg JS. Ganglionated plexus ablation vs linear ablation in patients undergoing pulmonary vein isolation for persistent/long-standing persistent atrial fibrillation: a randomized comparison. Heart Rhythm 2013;10:12806

10. Mamchur SE, Mamchur IN, Khomenko EA, Bokhan NS, Scherbinina DA. 'Electrical exclusion' of a critical myocardial mass by extended pulmonary vein antrum isolation for persistent atrial fibrillation treatment. Interv Med Appl Sci. 2014;6:31-9

11. Berger WR, Neefs J, van den Berg NWE, Krul SPJ, van Praag EM, Piersma FR, de Jong JSSG, van Boven WP, Driessen AHG, de Groot JR. Additional Ganglion Plexus Ablation During Thoracoscopic Surgical Ablation of Advanced Atrial Fibrillation: Intermediate Follow-Up of the AFACT Study. JACC Clin Electrophysiol. 2019;5:343-353

12. Mao J, Yin X, Zhang Y, Yan Q, Dong J, Ma C, Liu X. Ablation of epicardial ganglionated plexi increases atrial vulnerability to arrhythmias in dogs. Circ Arrhythm Electrophysiol. 2014;7:711-717

Table 1 Summary of randomized-controlled studies investigating the role of autonomic nervous system ablation in patients with atrial fibrillation 


\begin{tabular}{|c|c|c|c|c|c|c|}
\hline Author & $\begin{array}{l}\text { Patient } \\
\text { number }\end{array}$ & $\begin{array}{l}\text { Ablation } \\
\text { route }\end{array}$ & $\begin{array}{l}\text { GP } \\
\text { localization }\end{array}$ & Population & $\begin{array}{l}\text { Randomized } \\
\text { groups }\end{array}$ & $\begin{array}{l}\text { Outcomes } \\
\text { (AF/AT free } \\
\text { survival) }\end{array}$ \\
\hline Pokushalov $^{6}$ & 80 & Endo & $\begin{array}{l}\text { HFS or } \\
\text { Anatomical }\end{array}$ & PAF & $\begin{array}{l}\text { HFS } \\
\text { Anatomical }\end{array}$ & $\begin{array}{l}42.5 \% \text { in HFS } \\
77.5 \% \text { in } \\
\text { anatomical } \\
(\mathrm{p}=0.019)\end{array}$ \\
\hline Katritsis $^{7}$ & 67 & Endo & Anatomical & PAF & GP+PVI PVI & $\begin{array}{l}85.3 \% \text { in } \\
\text { GP+PVI } \\
60.6 \% \text { in PVI } \\
(\mathrm{p}=0.019)\end{array}$ \\
\hline Katritsis ${ }^{8}$ & 242 & Endo & Anatomical & PAF & $\begin{array}{l}\text { PVI GP } \\
\text { GP+PVI }\end{array}$ & $\begin{array}{l}56 \% \text { in PVI } \\
48 \% \text { in GP } \\
74 \% \text { in } \\
\text { GP+PVI (p = } \\
0.004)\end{array}$ \\
\hline Pokushalov $^{9}$ & 264 & Endo & Anatomical & $\begin{array}{l}\text { Per AF/ } \\
\text { LSPAF }\end{array}$ & $\begin{array}{l}\mathrm{PVI}+\mathrm{LL} \\
\mathrm{PVI}+\mathrm{GP}\end{array}$ & $\begin{array}{l}47 \% \text { PVI+LL } \\
54 \% \\
(\mathrm{PVI}+\mathrm{GP}) \\
(\mathrm{p}=0.29)\end{array}$ \\
\hline Mamchur $^{10}$ & 120 & Endo & Anatomical & $\begin{array}{l}\text { Per AF/ } \\
\text { LSPAF }\end{array}$ & $\begin{array}{l}\text { GP PVI } \\
\text { GP+PVI }\end{array}$ & $\begin{array}{l}38 \% \text { in GP } \\
56 \% \text { in PVI } \\
69 \% \text { in } \\
\text { GP+PVI (p } \\
=0.006 \text { for } \\
\text { GP+PVI vs } \\
\text { GP) }\end{array}$ \\
\hline Berger $^{11}$ & 240 & Thora & $\begin{array}{l}\text { HFS+ } \\
\text { anatomical }\end{array}$ & PAF/ Per AF & GP+PVI PVI & $\begin{array}{l}55.6 \% \text { in } \\
\text { GP+PVI } \\
56.1 \% \text { in PVI } \\
(\mathrm{p}=0.407)\end{array}$ \\
\hline Sandler 5 & 67 & Endo & HFS & PAF & GP PVI & $\begin{array}{l}49 \% \text { in GP } \\
61 \% \text { in PVI } \\
(\mathrm{p}=0.27)\end{array}$ \\
\hline
\end{tabular}

$A F$, atrial fibrillation; Endo, endocardial ablation; GP, ganglionated plexus ablation; HFS, high-frequency stimulation; LL, linear lesions; LSPAF, long-standing persistent atrial fibrillation; PAF, paroxysmal atrial fibrillation; Per AF, persistent atrial fibrillation; PVI, pulmonary vein isolation; Thora, thoracoscopic surgical ablation. 